\title{
Quantum power packs
}

a ow will you power a laptop quantum computer? With a quantum battery, obviously.

The scenario is hypothetical and might never come to pass, but it's not just a weak joke about some impending hegemony of quantumbased technologies. Quantum batteries are genuinely conceivable (although there's no reason to suppose their use would be limited to other quantum information technologies), and they propose to make use of quantum mechanics to improve the efficiency with which energy can be extracted from their charge reservoirs.

Loosely speaking, the notion of a quantum battery might be compared to the way quantum computers improve computational efficiency via entanglement of the quantum bits (qubits), leading to what some now call a 'quantum advantage' over classical devices. In that case, entanglement creates correlations between the states of the qubits. By the same token, Alicki and Fannes proposed that entangled copies of identical batteries - devices that can temporarily store energy in quantized energy states - might display gains in the maximal rate of extractable work ${ }^{1}$. They showed that the efficiency of energy extraction grows with the number of entangled copies.

Campaioli et al. subsequently calculated the upper bound on this collective 'quantum advantage' offered by an array of such quantum batteries, relative to equivalent energy-storage devices (such as capacitors) in which the charge/discharge events are independent ${ }^{2}$. Phrased this way, it sounds like a purely technical matter of making better batteries - ones, for instance, that can charge faster. But the significance goes deeper.

It is widely understood now that thermodynamics is closely connected to information theory, to the extent that information can itself be used as a kind of fuel to alter the energy of an object ${ }^{3}$. That's a corollary of the old idea, going back to Maxwell and his 'demon', that detailed information about a thermodynamic system can be used as a resource to do work, albeit without evading the second law.

Quantum batteries are in this respect, then, realizations of the same considerations about quantum information that underpin quantum computers, and which are extending ideas about classical thermodynamics into the quantum realm, where they have revitalized long-standing proposals of highly efficient quantum heat engines ${ }^{4}$.

Can quantum batteries actually be made, though? Ferraro et al. have now come up with a design that looks to be within the scope of current techniques ${ }^{5}$. Their idea is to make an array of solid-state devices with two distinct energy levels - these could, for example, be electronic states in a nanoelectronic device - that can be excited, as well as coupled and entangled together, via a single photonic mode in an optical cavity that contains the whole array.

The researchers compare this system with similar two-level devices

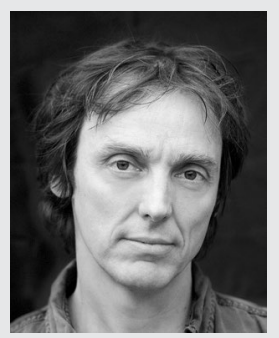

Philip Ball

that each have their own cavity and therefore act independently. They show that the charging power of the entangled array can be boosted (and thus the charging time decreased), relative to parallel charging of the independent storage units, by a factor that scales as the square root of the number of devices in the array. Ferraro et al. say that there exist several options for making two-level systems of this sort: using superconducting qubits, for example, or semiconducting quantum dots such as core-shell colloidal particles placed in an optical microcavity. It now seems quite feasible, then, to put these specific predictions of quantum advantage to the test.

Published online: 23 April 2018 https://doi.org/10.1038/s41563-018-0074-9

References

1. Alicki, R. \& Fannes, M. Phys. Rev. E 87, 042123 (2013).

2. Campaioli, F. et al. Phys. Rev. Lett. 118, 150601 (2017).

3. Toyabe, S., Sagawa, T., Ueda, M., Muneyuki, E. \& Sano, M. Nat. Phys. 6, 988-992 (2010).

4. Scovil, H. E. D. \& Schulz-DuBois, E. O. Phys. Rev. Lett. 2, 262-263 (1959).

5. Ferraro, D., Campisi, M., Andolina, G. M., Pellegrini, V. \& Polini, M. Phys. Rev. Lett. 120, 117702 (2018). 